\title{
ENSINO DE GERIATRIA E GERONTOLOGIA PARA O CURSO DE ODONTOLOGIA NA REGIÃO NORDESTE DO BRASIL
}

\author{
THE TEACHING OF GERIATRICS AND GERONTOLOGY TO A COURSE OF \\ DENTISTRY IN THE NORTHEAST REGION OF BRAZIL
}

\author{
Júlia Tavares Palmeira ${ }^{1}$, Vitor Nascimento Goes ${ }^{2}$, Waleska Fernanda Souto Nóbrega ${ }^{2}$, \\ Manuella Santos Carneiro Almeida ${ }^{3}$, Camila Helena Machado da Costa Figueiredo ${ }^{4}$
}

\section{RESUMO}

O objetivo desse trabalho foi verificar a presença da disciplina de geriatria na grade curricular dos cursos de odontologia nas universidades da região Nordeste do Brasil. Este estudo adotou como estratégia de coleta de dados a consulta no site do Ministério da Educação (MEC) e no Conselho Federal de Odontologia (CFO), por meio da visita dos sites dos cursos de odontologia. O universo foi composto por 95 universidades e após a aplicação dos critérios de inclusão e exclusão foi obtida uma amostra de 86 faculdades. Assim, das 86 faculdades consultadas, $45(52,32 \%)$ apresentaram a disciplina de geriatria, sendo $40(88,88 \%)$ em universidade particular e as outras $5(11,11 \%)$ em universidade pública. As outras 41 universidades $(47,67 \%)$ não apresentaram a disciplina. De acordo com resultados obtidos, pode-se concluir que foram encontrados um número relevante de faculdades que oferecem a disciplina de geriatria em odontologia na sua grade curricular.

Palavras-chave: Currículo, Educação, Educação em Odontologia, Geriatria, Gerontologia.

\section{ABSTRACT}

The objective of this work was to verify the presence of the geriatric discipline in the curriculum of dentistry courses in universities in the Northeast of Brazil. This study adopted as a data collection strategy the consultation on the website of the Ministry of Education (MEC) and the Federal Council of Dentistry (CFO), through the visit of the dentistry courses'websites. The universe consisted of 95 universities and after applying the inclusion and exclusion criteria, a sample of 86 colleges was obtained. Thus, of the 86 colleges consulted, $45(52.32 \%)$ presented the discipline of geriatrics, 40 (88.88\%) of them were private universities and the other 5 (11.11\%) were public universities. The other 41 universities (47.67\%) did not present the discipline. According to the results obtained, it can be concluded that a relevant number of colleges offering the subject of geriatrics in dentistry were found in their curriculum.

Keywords: Curriculum, Education, Dentistry Education, Geriatrics, Gerontology.

\footnotetext{
${ }^{1}$ Acadêmicos de Odontologia pela Universidade Federal de Campina Grande (UFCG). E-mails: julia.palmeira@hotmail. com; vitor9511goes@hotmail.com

${ }^{2}$ Mestre em Saúde Pública pela UEPB. E-mail: drawaleskasouto@gmail.com

${ }^{3}$ Professoras Doutoras da Universidade Federal de Campina Grande (UFCG). E-mails: manuellacarneiro@hotmail.com; camila_helena_@hotmail.com
} 


\section{INTRODUÇÃO}

Graças a melhorias no sistema de saúde, avanços tecnológicos, melhorias sociais e outros fatores que afetam positivamente a saúde e o bem-estar da população, tem se notado um aumento geral na expectativa de vida. Levantamentos feitos pelo Instituto Brasileiro de Geografia e Estatística (IBGE) apontaram que em 2018 a expectativa de vida do brasileiro foi de 76,3 anos, representando uma alta de 3 meses e 4 dias em comparação com 2017 (IBGE, 2018).

Com o aumento na expectativa de vida a população de idosos também aumenta e as políticas de saúde voltadas para esse grupo são desenvolvidas. Esse fator tem favorecido o desenvolvimento do mercado odontológico e os profissionais com capacitação para atendimentos odontogeriátricos se tornaram mais procurados. Odontogeriatria é uma especialidade da Odontologia que atua no atendimento aos pacientes da terceira idade, com ênfase nas atenções preventivas e curativas de pacientes com doenças e condições de caráter sistêmico e crônico (MOREIRA et al., 2014; FIUZA et al., 2013).

Com base nisso, torna-se necessário que durante a graduação de odontologia temas que abordem o funcionamento fisiológico e as alterações patológicas dos pacientes idosos sejam melhor desenvolvidos. Fatores como o vocabulário, os aspectos sociais e a comunicação acessível são essenciais para melhorar a relação profissional-paciente. Sem tal formação profissional, acaba-se por desrespeitar os fundamentos éticos da profissão de saúde, os quais englobam a oferta do melhor atendimento a todo e qualquer paciente (PORTO, 2018; CAPUTO et al., 2010)

Mesmo com a necessidade de formar cirurgiões-dentistas capacitados para saber lidar com esse público, a disciplina de odontogeriatria ou odontologia geriátrica ainda é bastante negligenciada na grade curricular das universidades, tendo seu conteúdo dissolvido juntamente a outras áreas do conhecimento. Desta forma, a defesa pela inclusão de disciplina específica evita que os conteúdos sobre saúde do idoso e envelhecimento sejam diluídos em outras disciplinas ou ministrados de forma parcial e possibilita contato mais aproximado do aluno com o idoso (NÚNEZ et al., 2016).

Visto a necessidade de aliar o envelhecimento da população com a demanda de saúde desse grupo, o objetivo desse trabalho é verificar a presença da disciplina de odontogeriatria na grade curricular dos cursos de odontologia nas universidades das regiões do Brasil.

\section{MATERIAL E MÉTODOS}

Este estudo foi do tipo transversal e descritivo, a coleta de dados foi realizada à partir da consulta de dados disponíveis no site do Ministério da Educação (MEC) e no Conselho Federal de Odontologia (CFO), por meio do acesso aos sites dos cursos de odontologia nas universidades da região Nordeste do Brasil. 
Foram incluídas na pesquisa todas as universidades que oferecem o curso de odontologia cadastrados no MEC ou no CFO, sendo instituições públicas ou privadas da região Nordeste do País. E foram excluídas da amostra todas as instituições de ensino superior que não disponibilizavam os seus componentes curriculares nos seus respectivos sites.

O universo foi composto por 95 universidades, contudo, após a aplicação dos critérios de inclusão e exclusão obteve-se uma amostra de 86 faculdades que dispuseram a grade curricular nos sites correspondentes.

A coleta de dados foi realizada por um pesquisador, que acessou o site do MEC e CFO arquivando o nome das universidades, os sites e os e-mails das IESs (Instituições de ensino Superior) que ofertam o curso de Odontologia na região Nordeste em uma tabela. De posse desses dados, o pesquisador localizou nos sites das IESs as matrizes curriculares, verificando a presença ou ausência da disciplina de odontogeriatria na grade curricular ou no plano de curso (PPC) e se esta era obrigatória ou optativa, também foi verificada a carga horária da disciplina.

Os dados coletados foram registrados na forma de banco de dados do programa de informática SPSS (Statistical Package for Social Sciences) para Windows, versão 13.0, e foram trabalhados pela descritiva.

\section{RESULTADOS}

A tabela 1 aponta o número de faculdades públicas e privadas da região Nordeste que fornecem a disciplina de odontogeriatria.

Tabela 1- Total de faculdades públicas e privadas da região Nordeste que fornecem a disciplina de odontogeriatria - 2020.

\begin{tabular}{|cccc}
\hline ESTADOS & Particular & Pública & Total por estado \\
\hline Alagoas & 3 & 0 & 5 \\
\hline Bahia & 12 & 2 & 25 \\
Ceará & 7 & 0 & 11 \\
\hline Maranhão & 3 & 0 & 8 \\
\hline Paraíba & 6 & 1 & 12 \\
\hline Pernambuco & 3 & 1 & 9 \\
\hline Piauí & 3 & 0 & 7 \\
\hline Rio Grande do Norte & 2 & 1 & 5 \\
\hline Sergipe & 1 & 0 & 86 \\
\hline TOTAL & 40 & 5 & \\
\hline
\end{tabular}

Assim, das 86 faculdades consultadas, 45 (52,32\%) apresentaram a disciplina de geriatria, sendo $40(88,88 \%)$ em universidade particular e as outras $5(11,11 \%)$ em universidade pública. As outras 41 universidades (47,67\%) não apresentaram a disciplina. 
Das instituições de ensino superior que ofertaram da disciplina em análise, 38 (84,44\%) são do tipo obrigatória e apenas 7 (15,55\%) são do tipo optativa, sendo que as cargas horárias variaram de 30 horas por semestre (Faculdade de Santa Maria) no estado da Paraíba a 160 horas (Faculdade de Ensino Superior da Cidade de Feira de Santana - FAESF) na Bahia. A carga horária citada com mais frequência foi correspondente a 60 horas (52,38\%) (Tabela 2).

Tabela 2- Divisão das faculdades (Pública/Privada) que possuem a disciplina de odontogeriatria (Optativa/Obrigatória) e suas respectivas cargas horárias- 2020.

\begin{tabular}{|c|c|c|c|}
\hline Instituição de Ensino Superior & $\begin{array}{l}\text { Pública/ } \\
\text { Privada }\end{array}$ & $\begin{array}{c}\text { Optativa/ } \\
\text { Obrigatória }\end{array}$ & $\begin{array}{c}\text { Carga } \\
\text { Horária }\end{array}$ \\
\hline Faculdade Ceuma - Uniceuma (MA) & Particular & Obrigatória & $80 \mathrm{hrs}$ \\
\hline Instituto Florence (MA) & Particular & Optativa & $40 \mathrm{hrs}$ \\
\hline Faculdade Maurício de Nassau - Uninassau (MA) & Particular & Obrigatória & $60 \mathrm{hrs}$ \\
\hline Escola Bahiana de Medicina e Saúde Pública (BA) & Particular & Obrigatória & $60 \mathrm{hrs}$ \\
\hline Faculdade de ensino superior de Floriano - FAESF (PI) & Particular & Obrigatória & $64 \mathrm{hrs}$ \\
\hline Centro universitário Uninovafapi (PI) & Particular & Obrigatória & $40 \mathrm{hrs}$ \\
\hline Faculdade Mauricio de Nassau - Uninassau (PI) & Particular & Obrigatória & $60 \mathrm{hrs}$ \\
\hline Fundação Edson Queiroz - UNIFOR (CE) & Particular & Optativa & - \\
\hline Faculdade metropolitana da grande Fortaleza (CE) & Particular & Obrigatória & $60 \mathrm{hrs}$ \\
\hline Centro universitário Christus - UNICHRISTUS(CE) & Particular & Obrigatória & $100 \mathrm{hrs}$ \\
\hline Centro universitário católica de Quixadá (CE) & Particular & Optativa & $60 \mathrm{hrs}$ \\
\hline Faculdade Mauricio de Nassau - Fortaleza (CE) & Particular & Obrigatória & $60 \mathrm{hrs}$ \\
\hline Faculdade Mauricio de Nassau - Juazeiro do Norte (CE) & Particular & Obrigatória & $60 \mathrm{hrs}$ \\
\hline Faculdade Mauricio de Nassau - Maracanaú (CE) & Particular & Obrigatória & $60 \mathrm{hrs}$ \\
\hline Universidade Federal de Pernambuco (PE) & Pública & Obrigatória & $120 \mathrm{hrs}$ \\
\hline Faculdade Mauricio de Nassau - Caruaru (PE) & Particular & Obrigatória & $60 \mathrm{hrs}$ \\
\hline Faculdade Mauricio de Nassau - Olinda (PE) & Particular & Obrigatória & $60 \mathrm{hrs}$ \\
\hline Faculdade Mauricio de Nassau - Recife (PE) & Particular & Obrigatória & $60 \mathrm{hrs}$ \\
\hline Universidade Estadual do Rio Grande do Norte (RN) & Pública & Optativa & $60 \mathrm{hrs}$ \\
\hline Faculdade Mauricio de Nassau - Parnamirim (RN) & Particular & Obrigatória & $60 \mathrm{hrs}$ \\
\hline Faculdade Mauricio de Nassau - Mossoró (RN) & Particular & Obrigatória & $60 \mathrm{hrs}$ \\
\hline Universidade Federal de Campina Grande (PB) & Pública & Obrigatória & $30 \mathrm{hrs}$ \\
\hline Instituto de Educação Superior da Paraíba - IESP (PB) & Particular & Obrigatória & $40 \mathrm{hrs}$ \\
\hline Faculdades Integradas de Patos - FIP (PB) & Particular & Obrigatória & - \\
\hline Faculdades Integradas de Patos - Campina Grande (PB) & Particular & Obrigatória & - \\
\hline Faculdade Santa Maria (PB) & Particular & Obrigatória & $30 \mathrm{hrs}$ \\
\hline Faculdade Rebouças (PB) & Particular & Obrigatória & - \\
\hline Faculdade Mauricio de Nassau - Campina Grande (PB) & Particular & Obrigatória & $60 \mathrm{hrs}$ \\
\hline Faculdade Mauricio de Nassau - Maceió (AL) & Particular & Obrigatória & $60 \mathrm{hrs}$ \\
\hline Faculdade Mauricio de Nassau - Arapiraca (AL) & Particular & Obrigatória & $60 \mathrm{hrs}$ \\
\hline Faculdade de Tecnologia de Alagoas (AL) & Particular & Obrigatória & - \\
\hline Faculdade Mauricio de Nassau - Sergipe (SE) & Particular & Obrigatória & $60 \mathrm{hrs}$ \\
\hline Universidade Federal da Bahia (BA) & Pública & Optativa & $68 \mathrm{hrs}$ \\
\hline Universidade Estadual Feira de Santana (BA) & Pública & Optativa & $60 \mathrm{hrs}$ \\
\hline Faculdade Mauricio de Nassau - Vitória da Conquista (BA) & Particular & Obrigatória & $60 \mathrm{hrs}$ \\
\hline Faculdade Mauricio de Nassau - Salvador (BA) & Particular & Obrigatória & $60 \mathrm{hrs}$ \\
\hline Faculdade Mauricio de Nassau - Lauro de Freitas (BA) & Particular & Obrigatória & $60 \mathrm{hrs}$ \\
\hline Faculdade Independente do Nordeste - FAINOR (BA) & Particular & Optativa & - \\
\hline
\end{tabular}




\begin{tabular}{|c|c|c|c|}
\hline UniFTC - Salvador (BA) & Particular & Obrigatória & $40 \mathrm{hrs}$ \\
\hline UniFTC- Vitória da Conquista (BA) & Particular & Obrigatória & $40 \mathrm{hrs}$ \\
\hline UniFTC Jequié (BA) & Particular & Obrigatória & $40 \mathrm{hrs}$ \\
\hline UniFTC Itabuna (BA) & Particular & Obrigatória & $40 \mathrm{hrs}$ \\
\hline UniFTC Feira de Santana (BA) & Particular & Obrigatória & $40 \mathrm{hrs}$ \\
\hline Faculdade de Ilhéus - Cesupi (BA) & Particular & Obrigatória & $72 \mathrm{hrs}$ \\
\hline Faculdade de Ensino Superior da Cidade de Feira de Santana - FAESF (BA) & Particular & Obrigatória & $160 \mathrm{hrs}$ \\
\hline
\end{tabular}

Fonte: Autoria própria

Das 45 faculdades listadas na tabela acima, a oferta da disciplina de estudo do tipo obrigatória varia desde o sétimo período ao décimo. A oferta em menor número corresponde ao sétimo período com apenas 1 (2,2\%) variando para o maior número que é o oitavo período com 26 (57,7\%).

Gráfico 1 - Distribuição da disciplina de acordo com o período que ela é ofertada

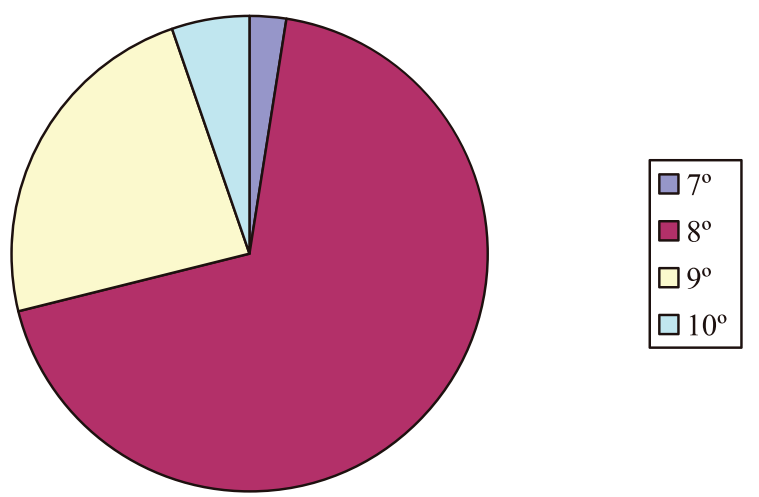

Fonte: Autoria própria

\section{DISCUSSÃO}

Segundo dados da World Health Organization (Who,2016) publicados em 2016 a expectativa de vida, a nível mundial, que era de 60 anos em 1970 tem crescido exponencialmente. Até o ano 2000 aumentou em 18,7 anos (78,7 anos) e em 2015 para 20,4 anos (80,4 anos). Este crescimento demográfico da população idosa tem sido alvo de grande interesse por parte da comunidade científica em vários países do mundo.

A geriatria, bem como a odontogeriatria, busca cada vez mais aumentar o tempo de vida do homem, mantendo-o preservado com relativa saúde, mas com alegria de viver, entendendo que a fase final da vida deve ser encarada como uma etapa que também tem seus encantos e que permite uma existência feliz e recompensadora (Montenegro et al., 2013).

Ao longo do processo de envelhecimento ocorrem tanto alterações físicas como sociais que podem comprometer o estado de saúde das pessoas, sendo por isso considerado um desafio a ser enfrentado pela sociedade (Miranda et al., 2016). Com o aumento da população idosa no mundo, a odontologia também está passando por diferentes desafios. Um deles é justamente a formação profissional daqueles que irão ser 
responsáveis pelo cuidado da população idosa. Neste contexto, torna-se relevante que haja, ao longo dessa formação, a possibilidade de contato com conhecimentos da área da geriatria e da gerontologia, como também o desenvolvimento de práticas de ensino adequadas, incluindo aqui as de saúde bucal (Ettinger, 2012).

O aumento das demandas nas áreas da prestação de serviços, pesquisa e políticas públicas, acompanha a realidade do crescimento da população idosa no Brasil. Assim, a formação de recursos humanos não pode ser desarticulada das necessidades existentes no país exigindo investimentos nesta formação e aprimoramento no estudo do envelhecimento. (Neri; Jorge, 2006)

Com o envelhecimento da população brasileira se faz necessário o aumento de cirurgiões-dentistas que conheçam e entendam as alterações características que acometem o organismo do idoso e suas consequências (Fernandes et al., 2016). Baseado no contexto do acadêmico estar inserido na capacitação no atendimento da faixa etária em estudo, a quantidade de faculdades que disponibilizam a disciplina mostrou um resultado relevante. Em todas as academias de odontologia consultadas, 45 $(52,32 \%)$ apresentaram a disciplina de geriatria de forma optativa/obrigatória.

Os achados deste estudo corroboram com evidências internacionais, os quais relatam que a disciplina de Odontogeriatria deve ser oferecida nos cursos de graduação e requer uma revisão profunda nos currículos atuais. A base para o currículo disciplinar em Odontogeriatria perpassa pela compreensão das características orais, médicas, psicológicas, físicas, nutricionais e mentais de pacientes idosos e deve estar pautado no cuidado centrado no paciente e na abordagem multidisciplinar e interdisciplinar, além de ultrapassar o aspecto eminentemente teórico e ser complementado com experiências práticas em diferentes situações (Kossioni et al., 2009).

Por se justificar em demasia, a necessidade do futuro profissional de atender o público idoso de forma correta e segura, nem todas as instituições de ensino que ofertam a disciplina são de caráter obrigatório. De todas faculdades listadas, 38 (84,44\%) são do tipo obrigatória e apenas 7 (15,55\%) são do tipo optativa. Além de toda a problemática existente na disponibilização da ciência em questão, o número de faculdades públicas que ofertam essa forma de conhecimento é praticamente inexpressiva. Do levantamento das 45 faculdades disponíveis, apenas $5(11,11 \%)$ universidades públicas dispõe de tal.

Observando a crescente expectativa de vida no contexto atual, surge a necessidade de se qualificar e quantificar a oferta desta disciplina, em termos metodológicos e de conteúdo, para que os futuros cirurgiões-dentistas estejam aptos a cuidar da saúde bucal dos idosos que estão em constante demanda. Nesse sentido, a inclusão da disciplina de odontogeriatria no currículo dos cursos pode ser uma possibilidade para uma melhor formação na área.

\section{CONCLUSÃO}

Diante dos resultados encontrados, pode-se concluir que um número relevante de faculdades que oferecem a disciplina de geriatria em odontologia foram encontradas. Um primeiro contato com 
a área de geriatria ainda na graduação é uma boa opção para impulsionar o aumento da quantidade de profissionais habilitados em realizar atendimentos com um maior compreendimento de toda complexidade psicossocial, pendências sistêmicas e alterações anatômicas que envolvem o público dessa faixa etária.

\section{REFERÊNCIAS}

CAPUTO, I. G. C. et al. Vidas em risco: Emergências Médicas em Consultório Odontológico. Rev Cir Traumatol Buco-Maxilo-Fac, v. 10, n. 3, p. 51-58, 2010.

ETTINGER, R. L. A 30-year review of a geriatric dentistry teaching programme. Gerodontology. v. 29, n. 2, p. 1252-1260, 2012.

FERNANDES NETO, J. A.; SILVA, A. M. T.; CATÃO. M. H. C. V. Odontogeriatras, geriatras e idosos brasileiros: uma análise por estados e regiões do país. Arch Health Invest, v. 5, n. 5, p. 262-266, 2016.

FIUZA, M. K. et al. Avaliação da prevalência e do grau de conhecimento do cirurgião-dentista em relação às emergências médicas. RFO. v. 18, n. 3, p. 295-301, 2013.

IBGE - Instituto Brasileiro de Geografia e Estatística. Censo Demográfico, 2018. Disponível em: https://glo.bo/3ehRZ4n. Acesso em: 01 de mar. 2020.

KOSSIONI, A. et al. undergraduate curriculum guidelines in Gerodontology. Gerodontology. Heath R. European College of Gerodontology v. 26, n. 3, p. 165-171, 2009.

MONTENEGRO, F. L. B.; MARCHINI, L. Odontogeriatria uma visão gerontológica. Rio de Janeiro: Elsevier. v. 3, n. 2, p. 1-360, 2013.

MIRANDA, G. M. D.; MENDES, A. C. G.; SILVA, A. L. A. O envelhecimento populacional brasileiro: desafios e consequências sociais atuais e futuras. Rev Bras Geriatr Gerontol. v. 16, n. 3, p. 507-519, 2016.

MOREIRA, A. C. L. et al. OS 15 ANOS DO PROJETO SORRISO GRISALHO E OS AVANÇOS NO CAMPO DA ODONTOGERIATRIA. Extensão em Ação. v. 2, n. 7, p. 97-110, 2014. 
NERI, A. L.; JORGE, M. D. Atitudes e conhecimentos em relação à velhice em estudantes de graduação em educação e em saúde: subsídios ao planejamento curricular. Revista estudos de Psicologia. v. 23 , n. 2, p. 127-137, 2006.

NÚNEZ, M. R. R.; GODOI, H.; MELLO, A. L. S. F. Panorama do ensino de odontogeriatria nas universidades públicas brasileiras. Revista Família, Ciclos de Vida e Saúde no Contexto Social. v. 4, n. 3, p. 237-245, 2016.

PORTO, É. Et al. Ensino da Odontogeriatria em faculdades de Odontologia da região Nordeste do Brasil. Arch Health Invest. v. 7, n. 2, p. 48-50, 2018.

World Health Organization (WHO). World health Statistics: monitoring health for the sustainable development goals. 2016. 